\title{
VECTOR ANALYSIS OF THE rS PATTERN IN LEAD V5
}

\author{
BY \\ P. SCHWEITZER \\ From the Medical Department, Medical Faculty of the Šafárik's University Košice, Czechoslovakia
}

Received February 26, 1961

A small $\mathrm{r}$ wave and a deep $\mathrm{S}$ wave in lead V5 (rS pattern) are observed in hypertrophy of the right ventricle (Myers et al., 1948), in clockwise rotation of the heart (Goldberger, 1953), and in bilateral hypertrophy of the ventricles (Pagnoni and Goodwin, 1952). Anterior cardiac infarction may also be a cause (Myers and Klein, 1949; Levy and Hyman, 1950). An rS pattern has been observed by Leatham (1950) in 2 of 100 healthy persons; in one subject, the $R$ and $S$ waves were identical. Goodwin (1958) observed an rS pattern in 85 patients with morbid anatomical findings; 51 showed right or combined ventricular hypertrophy. Myocardial infarction was present in the remaining 34; it was combined 26 times with univentricular or biventricular hypertrophy of one or both ventricles. In 8 patients, isolated myocardial infarction was observed.

In view of the fact that the usual electrocardiographic analysis cannot always differentiate these lesions, an attempt is here made to determine the value of the vector analysis in the correct interpretation of the $\mathrm{rS}$ pattern in lead V5.

\section{SubJECTS AND METHODS}

Twenty-six patients were examined in whom an rS pattern in lead V5 was found 25 times; in one instance both waves were identical. In 20 patients the vectorcardiogram (VCG) was compared with the pathological findings. The loop was derived in the frontal plane from leads aVF and aVL, in the horizontal plane from leads V1 and V6. In each patient, six leads from the extremities and six præcordial leads were taken. A thickness of more than $15 \mathrm{~mm}$. of the left ventricular wall or if more than $5 \mathrm{~mm}$. of the right ventricular wall were accepted as criteria of ventricular hypertrophy.

\section{RESULTS}

The patients were divided into three groups according to the clinical picture and autopsy findings.

1. Right ventricular hypertrophy: 11 patients.

2. Bilateral ventricular hypertrophy: 9 patients.

3. Myocardial infarction: 6 patients.

1. Right Ventricular Hypertrophy. In this group isolated right ventricular hypertrophy was proven at necropsy in 8 patients, while in 3 others, the diagnosis was established with the aid of clinical and laboratory findings.

Vectorcardiogram. Frontal plane. Loops in this plane exhibit approximately identical form and direction (Fig. 1). They begin to the left, some of them superiorly, some inferiorly. Thereafter. the loop takes a right downwards turn. In 10 cases the maximal vector is located to the right and in one case to the left and inferiorly. In 9 patients the loop is inscribed in a clockwise direction and 


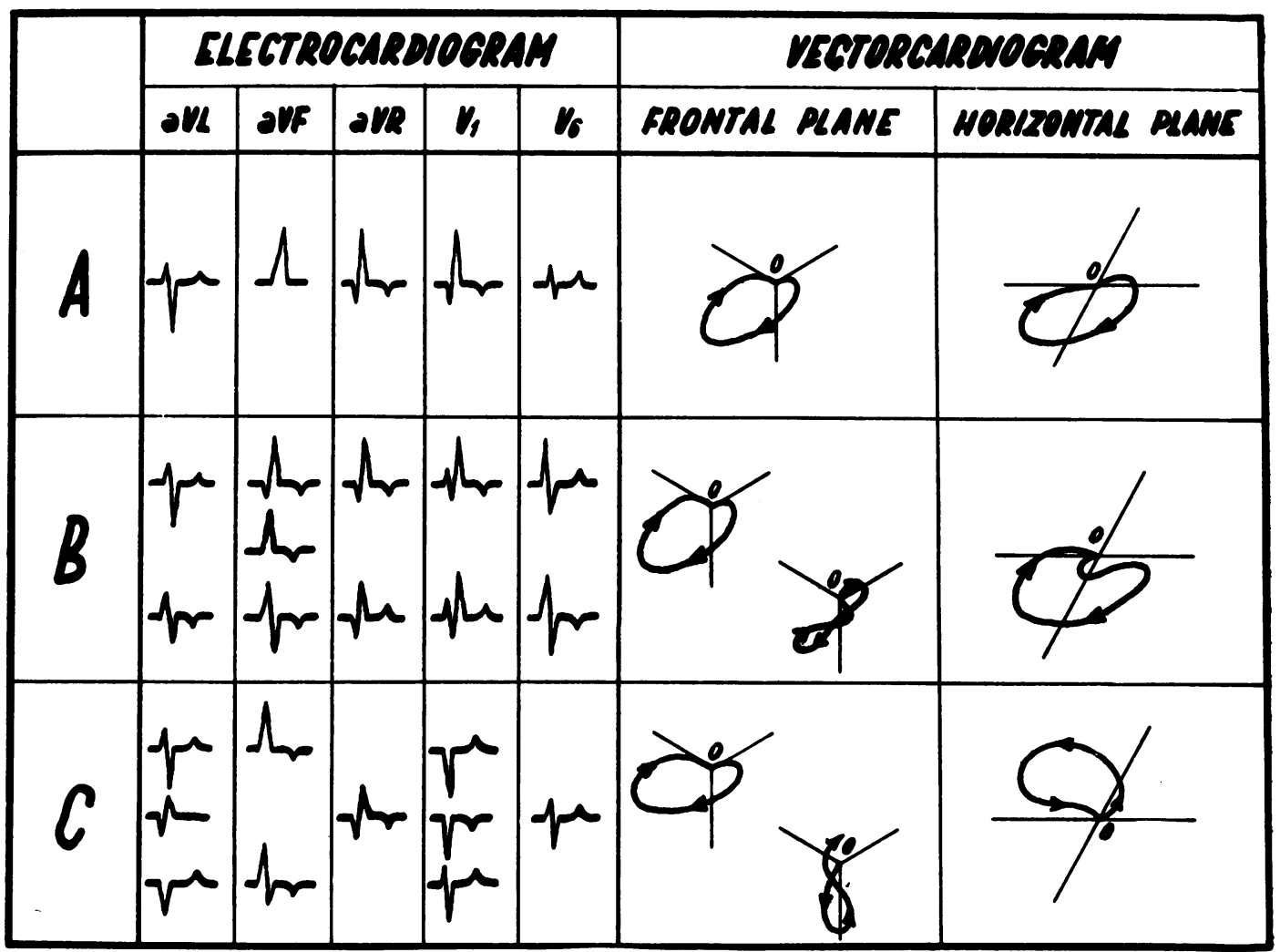

FIG. 1.-Electro- and vector- cardiogram in patients with hypertrophy of the right ventricle. For description see text.

in two it is very narrow and presents a "figure of eight" configuration. The $T$ vector is directed to the left and superiorly, only in two cases to the left inferiorly; it is always directed in a sense opposite to the maximal vector.

Horizontal plane. Loops in this plane may be subdivided in three groups (Fig. 1A, 1B, 1C).

In the first are two patients with right ventricular hypertrophy proven at autopsy, the loop being oriented posteriorly and to the left (Fig. 1A); thereafter, it takes an anterior direction and finally to the right. The loop is inscribed in a clockwise direction. The maximal vector is located in the first case anteriorly and in the latter to the left. In both the $T$ vector is directed to the left. The angle between the maximal and the $T$ vector is greater than $45^{\circ}$.

In 4 other patients (Fig. 1B) with right ventricular hypertrophy, the loop begins anteriorly to the right and in the middle part to the left, whereas the terminal part is orientated anteriorly and to the right. The loop is inscribed in a clockwise direction. The maximal vector was located three times anteriorly and to the right, once to the right and posteriorly. The $T$ vector is always located posteriorly, the angle between the $\mathrm{T}$ vector and the maximal vector being approximately $180^{\circ}$.

In the third group, there were 5 patients with chronic pulmonary heart disease. Right ventricular hypertrophy was proven at necropsy in two (Fig. 1C and Fig. 2).

The initial portion of the loop is displaced posteriorly and to the left or posteriorly and only exceptionally to the right and anteriorly. The greater part of the loop is orientated in the right and posterior segment. The loop is inscribed in a counter-clockwise direction and the maximal vector located posteriorly and to the right. The vector of the $T$ wave is directed to the left. The angle between them is greater than $90^{\circ}$. 


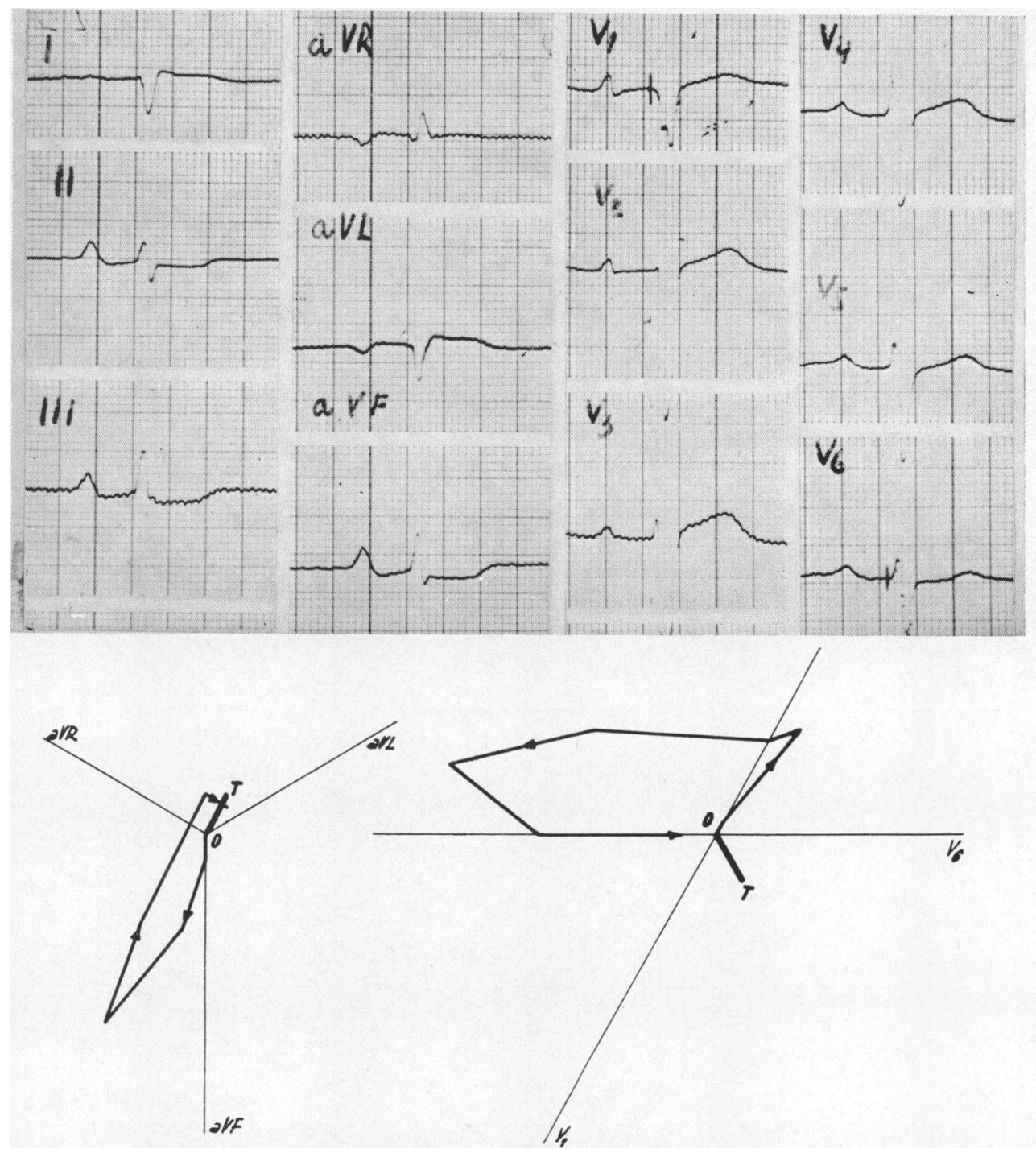

FIG. 2.-(Case 7). Electro- and vector-cardiograms of patient with lone right ventricular hypertrophy. For description, see text.

Electrocardiogram. First group (Fig. 1A). In both patients a vertical heart position is observed, in the aVR and V1 leads, the QRS complex has a qR form, and in lead V6 an rS form.

Second group (Fig. 1B). Vertical heart position. In three patients lead aVR had the form of $\mathrm{qR}$ and in one patient qr. In V1 and V2 an rSR form is found and in lead V6 an rS pattern.

Third group (Fig. 1C). Vertical heart position. In lead aVR the QRS complex is 4 times $q R$ and once QS; in leads V1 and V2 the QS form is seen in three patients; in two others rS. In lead V6 there is an $\mathrm{rS}$ pattern.

2. Bilateral Ventricular Hypertrophy. Combined ventricular hypertrophy was proven at necropsy in seven cases of this group. In the remaining two, there was a combination of ischæmic heart disease with chronic cor pulmonale. In Fig. 3 the electro- and vector-cardiograms and necropsy findings are presented.

Vectorcardiogram. Frontal plane. In four patients the loop is inscribed in a clockwise and in one 


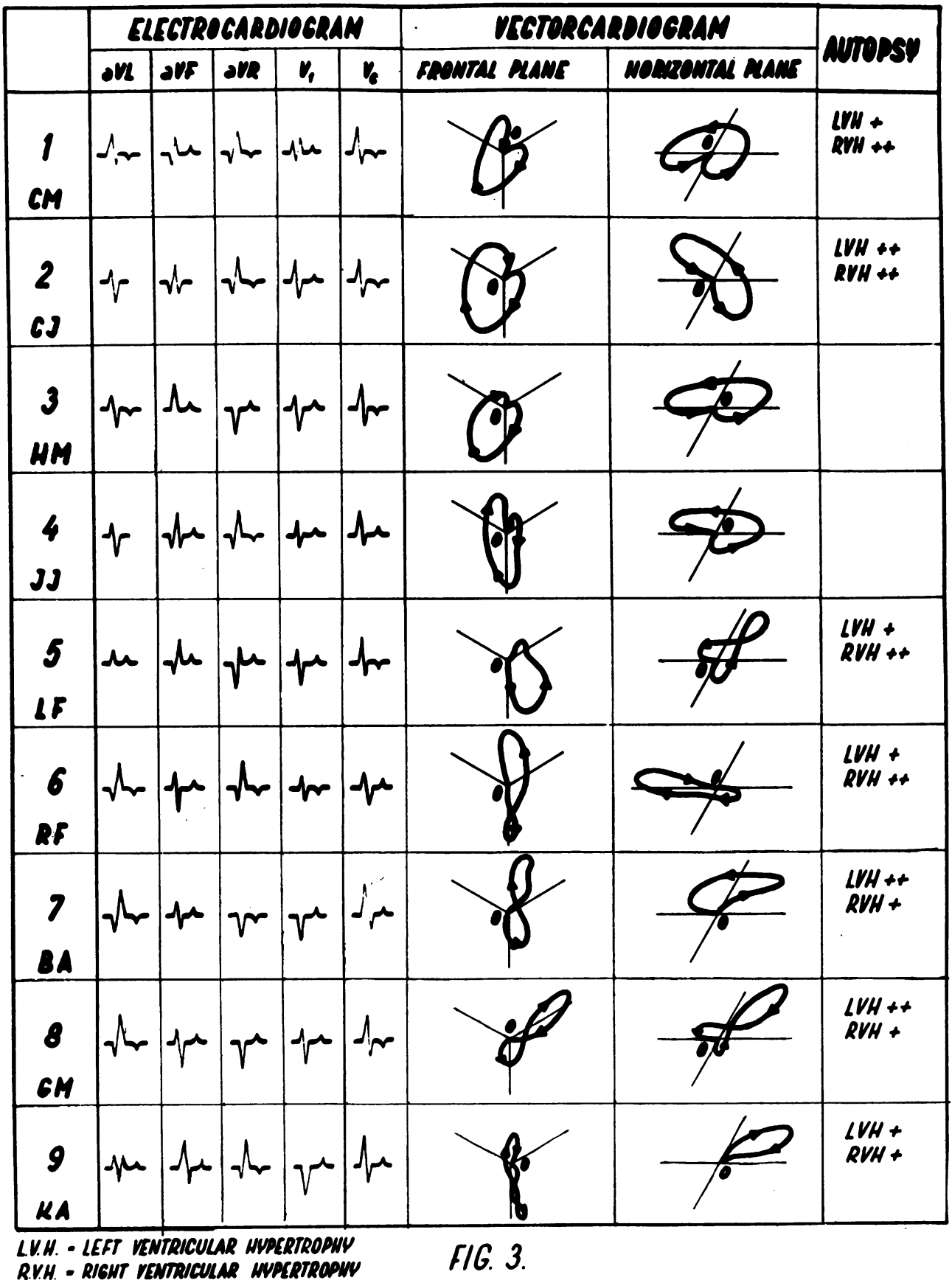

FIG. 3.-Electro- and vector-cardiograms, and the necropsy findings in patients with combined ventricular hypertrophy. 

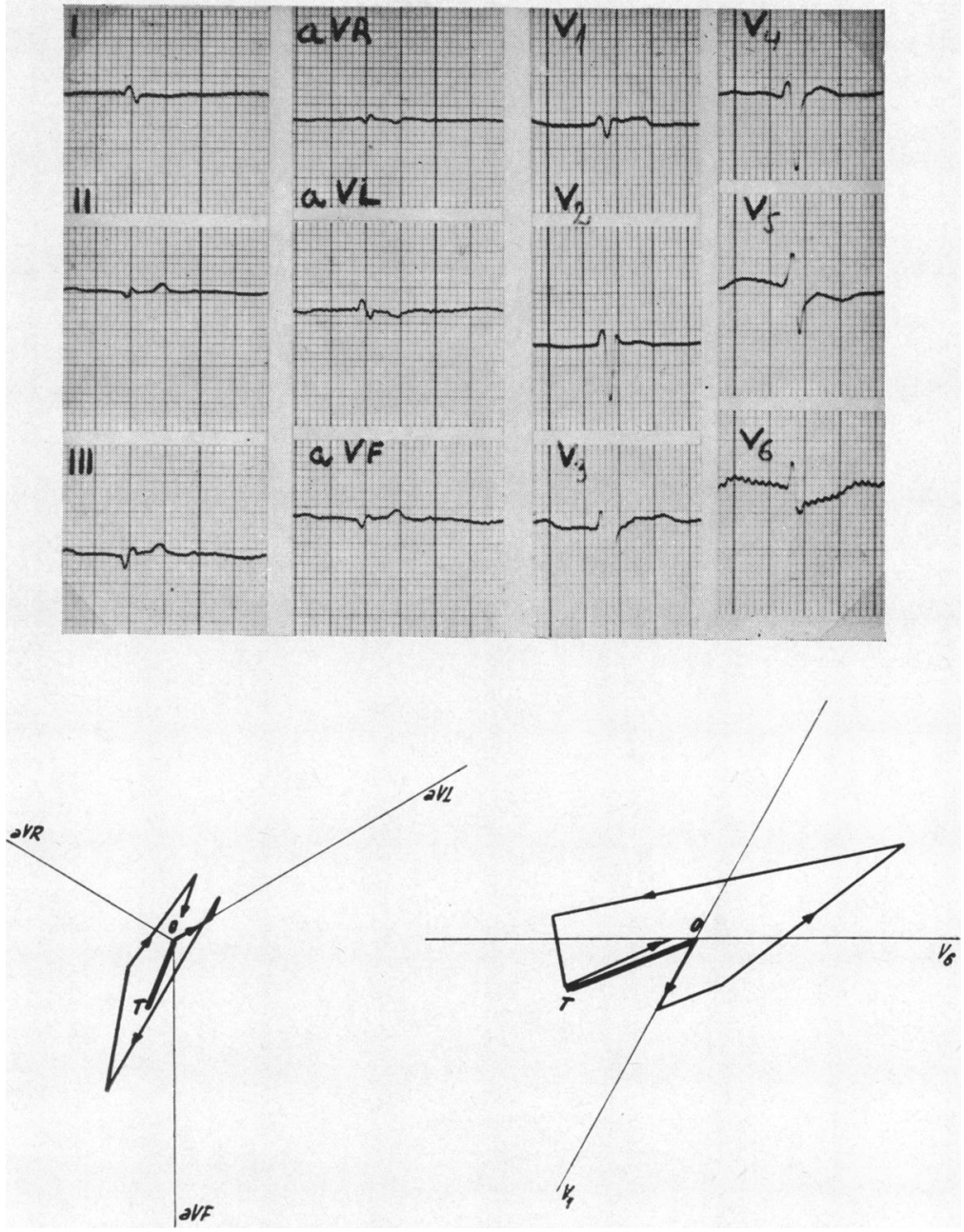

Frg. 4.-(Case C.M.). Electro- and vector-cardiograms or patient with combined ventricular hypertrophy. ECG: in extremity leads low voltage. QRS complex in III lead shows the form of qr and positive $\mathrm{T}$ wave. In V1 rs, in V2 rSŕ. In V6 RS and $\mathrm{S}-\mathrm{T}$ segment depression. VCG: in the frontal plane the loop starts to the left and superiorly, turning then inferiorly and to the right, where the maximal vector also tends. $T$ vector is located inferiorly and to the right. In the horizontal plane the loop is inscribed in the counterclockwise direction first at all anteriorly, then posteriorly. The terminal part is orientated anteriorly and to the right.

in a counter-clockwise direction: the maximal vector is located inferiorly and sometimes to the right. The $\mathrm{T}$ vector is three times directed in the sense of the maximal vector, twice in the opposite sense. In case 6 the counterclockwise direction of inscription of the VCG loop is observed and the maximal vector is located superiorly. The remaining three patients have the loop in the "figure of eight" configuration. Two of them had the maximal vector located upwards and to the left; in one it is downwards and to the left. The T-wave vector is directed superiorly and to the left.

Horizontal plane. In this projection, in Cases 1, 2, 3, 4, and 7, the VCG loop begins anteriorly, and thereafter turns to the left and finally posteriorly to the right. The loop is inscribed in a counterclockwise direction. The vector of the $T$ wave is orientated twice to the left, anteriorly and in 

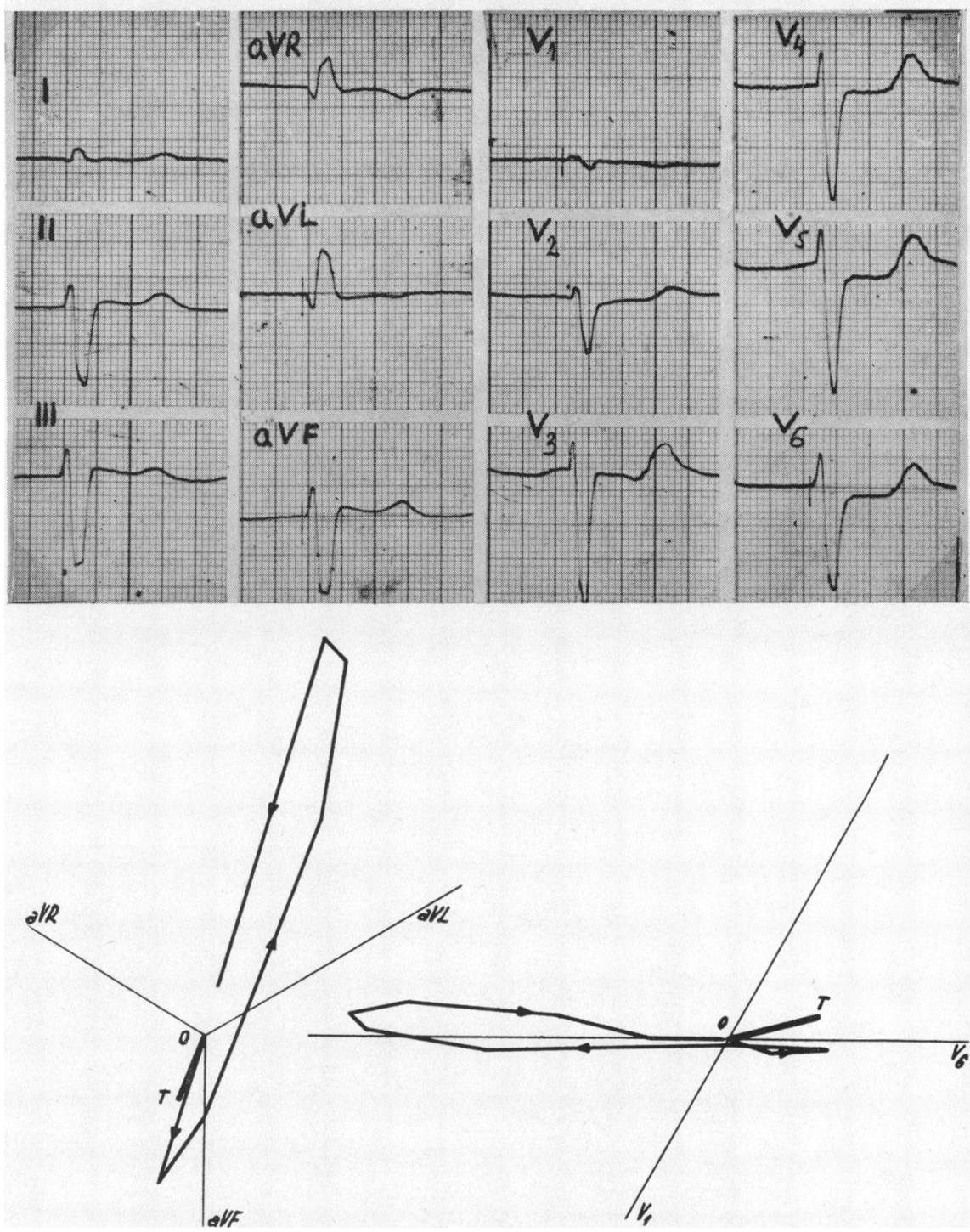

FIG. 5.-(Case R.F.). Electro- and vector-cardiograms of patient with combined ventricular hypertrophy. The cardiogram shows a horizontal position of the heart with deep SII and SIII waves. In lead $\mathrm{aVR}$ is $\mathrm{a} \mathrm{qR}$ and a negative $\mathrm{T}$ wave. In lead $\mathrm{V} 1, \mathrm{rS}$ and negative $\mathrm{T}$ wave. In $\mathrm{V} 6$ is $\mathrm{rS}$, with $\mathrm{S}-\mathrm{T}$ segment depression and a positive T wave. VCG: frontal plane-the loop is inscribed in counter-clockwise direction. The maximal vector is located superiorly and to the left, the $\mathrm{T}$ vector inferiorly and to the right. Horizontal plane: the loop is inscribed in a clockwise direction. The $T$ vector is located to the left and posteriorly.

three other instances to the right and anteriorly (Fig. 4). In two other patients (Cases 5 and 8) the loop has a "figure of eight" configuration and its distal part is inscribed in a counterclockwise direction and orientated in the left and posterior segment. The maximal vector is located to the left and posteriorly, the $T$ vector anteriorly and to the right. The loop of Case 8 (Fig. 5) begins anteriorly and to the left, and thereafter turns in a clockwise direction to the right and posteriorly. The maximal vector is inscribed to the right and posteriorly, the $T$ vector to the left and posteriorly. In 
the last patient of this group (K.A.) the loop begins to the left and posteriorly, thereafter in the clockwise sense anteriorly and finally regresses to the zero point. The maximal vector is directed to the left and posteriorly, and the $\mathrm{T}$ vector to the right and anteriorly.

Electrocardiogram. In 5 patients we found a vertical lie of the heart, in one an intermediate position, and in three a horizontal one.

Lead aVR: in 5 patients we found a $\mathrm{qR}$ with a negative $\mathrm{T}$ wave; in 3 patients $\mathrm{qS}$. In these the $\mathrm{T}$ wave was twice positive, and once negative. In one the configuration of the QRS complex was $\mathrm{Qr}$ and the $\mathrm{T}$ wave was positive.

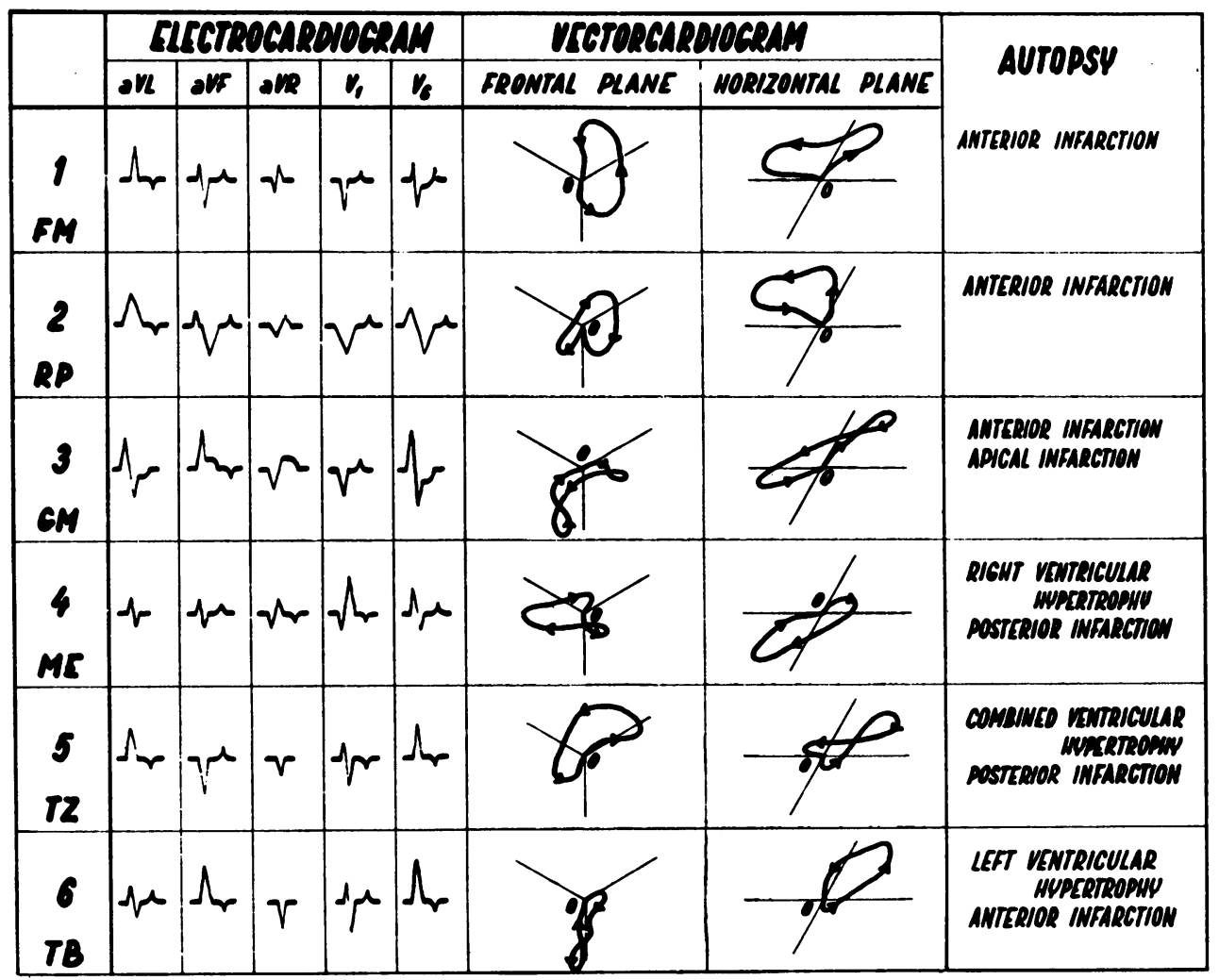

FI6. 6.

FIG. 6.-Electro- and vector-cardiograms, and necropsy findings in patients with myocardial infarction, with or without hypertrophy of one of the ventricles.

Lead V1: in one patient we have recorded rSR and a positive T wave; five patients had a QRS complex of $\mathrm{rS}$ form. In four patients the $\mathrm{T}$ wave was negative, in one positive. In this lead a QS with a positive $T$ wave was seen in two patients. One patient had an RS pattern and a positive T wave.

3. Myocardial Infarction. In Fig. 6 the electro- and vector-cardiograms and necropsy findings of the patients of this group are presented. These six patients may be divided in two sub-groups: in the first, there are three with anterior infarction, and in the second three patients where myocardial infarction was combined with ventricular hypertrophy.

A. Patients with Anterior Myocardial Infarction. Vectorcardiogram. Frontal plane. In Case 1 (Fig, 7) the loop is inscribed in a counter-clockwise direction and in Case 2 in a clockwise direction. The maximal vector in both patients is located superiorly and to the left, the $\mathrm{T}$ vector 

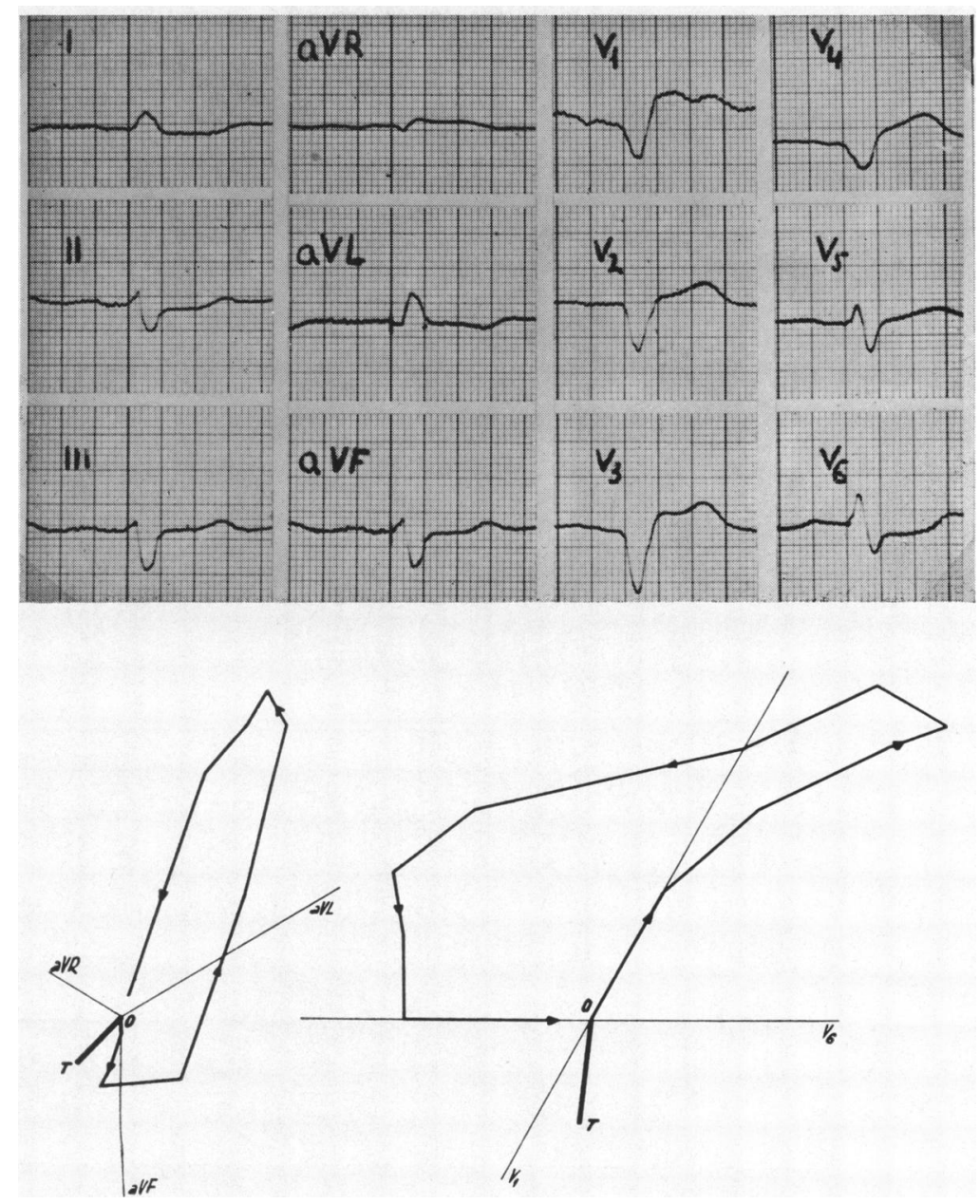

FIG. 7.-(Case 1). Electro- and vector-cardiograms, of patient with anterior myocardial infarction. For description, see text.

in the opposite direction. In Case 3 the initial portion of the VCG loop is displaced to the left, and the remainder of the loop is of "figure of eight" configuration. The maximal vector is located inferiorly and to the right, the $T$ vector superiorly and to the left.

Horizontal plane. The orientation and form of the VCG loop in Cases 1 and 2 are the same. The loop begins posteriorly, somewhat to the left and then passes clockwise to the right. The maximal vector is located posteriorly and to the right, the $T$ vector anteriorly and to the left. In Case 3 the loop has a "figure of eight" configuration; its terminal portion is located to the right and anteriorly, and the $\mathrm{T}$ vector is directed to the right and anteriorly.

Electrocardiogram. In the first two patients the heart was horizontal in position. In lead aVR the QRS complex was formed as a $\mathrm{qR}$, and in V1-4 as QS while the T wave was positive. In lead V6 there was an RS form and a depression of the S-T segment. In Case 2 there was left bundle-branch block. In the third patient, the heart was intermediate in position. The S-T segment in leads III, aVF, and aVR was elevated. In leads I and aVL the S-T segment was depressed. In lead V1 we found a QS form and a positive T wave; in lead V6 an RS was present and a depression of the S-T segment. 
B. Patients with Myocardial Infraction and Ventricular Hypertrophy. Case 4. Clinical diagnosis: chronic pulmonary heart disease. Necropsy findings: right ventricular hypertrophy and posterior myocardial infarction.

Vectorcardiogram. Frontal plane. The loop begins inferiorly and to the left inscribing a "figure of eight." Thereafter the loop turns to the right and returns in a clockwise direction towards the zero point. The maximal vector is directed to the right, and the $T$ vector downwards.

Horizontal plane. The loop begins posteriorly, and is thereafter directed anteriorly and finally to the right. The maximal vector is located to the right and anteriorly, the $T$ vector posteriorly and to the left.

Electrocardiogram. The heart was vertical in position. In aVR and V1 leads were found qR forms with negative $\mathrm{T}$ waves. In V6 lead an $\mathrm{rS}$ was combined with depression of the $\mathrm{S}-\mathrm{T}$ segment.

Case 5. Clinical diagnosis: hypertension, posterior myocardial infarction. Necropsy findings: combined ventricular hypertrophy, posterior myocardial infarction, repeated pulmonary embolism.

Vectorcardiogram. Frontal plane. The loop is characterized by an initial deflection to the left, thereafter turning to the right. The loop is inscribed in a counter-clockwise direction. The maximal vector is located superiorly and to the left, with the $T$ vector inferiorly.

Horizontal plane. At the beginning the loop is directed anteriorly, then posteriorly and to the left, where a "figure of eight" form is seen. The centripetal portion of the loop is located somewhat to the left, the maximal vector posteriorly and to the left, and the $T$ vector to right and posteriorly.

Electrocardiogram. The heart was horizontal in position. In lead V1 the QRS complex had the rS form and a negative $T$ wave. In lead V6 the QRS complex is formed from the $R$ wave and a negative $T$ wave.

Case 6. Clinical diagnosis: aortic regurgitation, congestive heart failure. Necropsy findings: left ventricular hypertrophy, anterior myocardial infarction.

Vectorcardiogram. Frontal plane. After a small initial deflection superiorly the loop turns inferiorly and somewhat to the right. The loop is inscribed in a clockwise direction; the maximal vector is located inferiorly and the $T$ vector superiorly and to the left.

Horizontal plane. The loop begins anteriorly, and then turns to the left and posteriorly. The maximal vector is directed posteriorly, the $\mathrm{T}$ vector anteriorly and to the right.

Electrocardiogram. The heart was vertical in position. In leads V1-5 there was a low $\mathrm{r}$ wave with a deep and broad $\mathbf{S}$ wave; $\mathbf{T}$ waves are negative. In lead $\mathrm{V} 6$ there is an $\mathbf{R}$ wave with a negative $\mathbf{T}$ wave.

\section{Discussion}

In patients with the rS pattern in V5 right ventricular hypertrophy is usually found. The vectorcardiographic pattern in the frontal plane in these patients is almost identical. In the horizontal projection, three types of vector loops can be identified. In the first type (Fig. 1A) the initial portion of the loop is displaced to the left and posteriorly. Then the loop turns anteriorly and to the right. The VCG of this type has been described by Fowler and Helm (1953), Pipberger et al. (1955) found those types in initial stages of right ventricular hypertrophy. The second pattern corresponds to the first type according to Grischman and Scherlis (1952). In five patients the initial portion of the loop was displaced posteriorly, but exceptionally anteriorly and to the right. The greater portion of the loop is situated in the right and posterior segment. These loops deserve our attention because besides the right ventricular hypertrophy they may present a manifestation of an anterior myocardial infarction (Stern and Tenney, 1956). The differential diagnosis between right ventricular hypertrophy and an anterior infarction is possible in the frontal plane. In patients with right ventricular hypertrophy, the loop is orientated in a clockwise sense and the maximal vector is located inferiorly and to the right. In an anterior myocardial infarction the loop is inscribed in a counterclockwise direction and the maximal vector is directed to the left and upwards. This observation is in accordance with the opinion of Goodwin (1958), who believes, that a vertical heart position is not encountered with myocardial infarction. However, in our series we observed one patient in whom the heart was vertical in spite of myocardial infarction and left ventricular hypertrophy.

A correct diagnosis in combined ventricular hypertrophy is very difficult to establish. In five patients of group 2 the loop in the horizontal plane was situated not only in the posterior and left segment, but also in the posterior and right one. In these vectorcardiograms the terminal portion of the loop could be regarded as representing the activation of the right ventricle (Wenger, 1956). 
Further evidence which could evoke a suspicion of bilateral ventricular hypertrophy, is a discrepancy between the forms of the VCG loop in the horizontal plane and in the frontal plane. In the first group (right ventricular hypertrophy) it was observed that if in the horizontal plane signs of right ventricular hypertrophy could be seen, then the vector loop in the frontal plane verified the diagnosis. In Case 6 with combined hypertrophy sings of right ventricular hypertrophy were found in the horizontal plane, while the loop in the frontal plane suggested rather a lesion of the left heart. The opposite can be observed in Cases 5 and 9, in which with a vertical heart position a conduction delay in the left bundle-branch was established.

To prove the diagnostic value of the discrepancy between the loops in the horizontal and frontal plane in combined ventricular hypertrophy it is necessary to examine a greater number of patients.

The diagnostic value of vectorcardiography in patients with an $\mathrm{rS}$ in $\mathrm{V} 5$ in myocardial infarction is very small. Among three patients with myocardial infarction in two both cardiogram and VCG led to the suspicion of a myocardial infarction. In the third, there was elevation of the S-T segment in aVF and in lead III, which strongly suggested a posterior infarction; but changes in the QRS complex and in the VCG loop were not characteristic of a myocardial infarction. In three other patients, where a myocardial infarction combined with ventricular hypertrophy was found, the diagnostic evidence was as follows. In Case 4(Fig. 6) with posterior infarction and right ventricular hypertrophy electro- and vector-cardiographic changes proved only right ventricular hypertrophy. In Case 5 (Fig. 6) cardiography and VCG indicated a posterior myocardial infarction. The loop in the horizontal plane in the last patient (Fig. 6) is characteristic of hypertrophy of the left ventricle. Higher voltage of the $\mathrm{R}$ wave in V4 and V5 was not present. This fact may be explained by the absence of hypertrophy of the left ventricle, or the hypertrophy might have been present in combination with an anterior infarction.

\section{SUMMARY AND CONCLUSION}

Derived vectorcardiography has been performed in 25 patients with the rS pattern in V5. In one patient $R$ and $S$ waves were identical. In 20 patients the vector-cardiographic patterns were correlated with necropsy findings. The rS pattern was observed in right ventricular hypertrophy, combined ventricular hypertrophy and in myocardial infarction, which latter may be (but not necessarily) associated with ventricular hypertrophy.

The vectorcardiographic analysis of the rS pattern in V5 renders it possible to differentiate right ventricular hypertrophy from the other conditions. Its value in the differential diagnosis of combined ventricular hypertrophy is smaller. This latter diagnosis should always be borne in mind if the central and terminal portions of the loop in the horizontal plane are displaced somewhat to the right and to the posterior segment. Similarly, attention should be given to the discrepancies between the form of the loop in the horizontal and in the frontal planes. According to our experience the value of vectorcardiography in the diagnosis of myocardial infarction is very small, especially in comparison with electrocardiography.

\section{REFERENCES}

Fowler, N. O., and Helm, R. A. (1953). Circulation, 7, 573.

Goldberger, E. (1953). Unipolar Lead Electrocardiography and Vector Cardiography. Lea and Febiger, Philadelphia. Goodwin, J. F. (1958). Brit. Heart J., 20, 191.

Grischaman, A., and Scherlis, L. (1952). Spatial Vectorcardiography. W. B. Saunders Co. Philadelphia.

Leatham, A. (1950). Brit. Heart J., 12, 213.

Levy, L. and Hyman, A. L. (1950). Amer. Heart J., 39, 243.

Myers, G. B., and Klein, H. A. (1949). Amer. Heart J., 37, 374.

,-- , and Stofer, B. E. (1948). Amer. Heart J., 35, 1.

Pagnoni, A., and Goodwin, J. F. (1952). Brit. Heart J., 14, 785.

Pipberger, H., Luchsinger, P., Kalin, R., and Schaub, F. (1955). Cardiologia, 27, 65.

Stern, E. A., and Tenney, S. M. (1956). Amer. Heart J., 51, 53.

Wenger, R. (1956). Klinische Vectorkardiographie. Steinkopff, Darmstadt. 